\title{
Intranasal Delivery of Human Wharton's Jelly- Derived Mesenchymal Stem Cells Alleviate Memory Deficits In Sporadic Alzheimer's Rat Model By Regulating Neurotrophic and Apoptosis Genes
}

\section{Ebrahim Eslami}

Islamic Azad University of Tehran: Islamic Azad University Central Tehran Branch

Farshid Qiyami-Hour

IUMS: Iran University of Medical Sciences

Marjan Sadr

Islamic Azad University of Tehran: Islamic Azad University Central Tehran Branch

Marziyeh Ajdary

IUMS: Iran University of Medical Sciences

Ronak Shabani

IUMS: Iran University of Medical Sciences

Mehdi Mehdizadeh ( $\square$ mehdizadeh.m@iums.ac.ir)

Iran University of Medical Sciences https://orcid.org/0000-0002-9268-7318

\section{Research Article}

Keywords: Alzheimer's model, mesenchymal stem cells, wharton's jelly, neurotrophic factors, apoptotic factors

Posted Date: April 14th, 2021

DOI: https://doi.org/10.21203/rs.3.rs-408910/v1

License: (a) (i) This work is licensed under a Creative Commons Attribution 4.0 International License. Read Full License 


\section{Abstract}

Alzheimer's disease (AD) is the most common cause of dementia in adulthood, which is followed by cognitive impairment and behavioral deficits. Today, mesenchymal stem cell (MSC)-based therapy is a good therapeutic option to improve regenerative medicine in neurodegenerative disorders including AD. The aim of this study is to investigate the effects of the human Wharton's jelly-derived MSCs (WJ-MSCs) on Alzheimer's rat models by studying the expression of neurotrophic factors involved in neurodegenerative diseases such as brain-derived neurotrophic factor (BDNF) and nerve growth factor $(N G F)$, as well as expression of apoptotic factors such as B-cell lymphoma 2 (BCL2, apoptosis inhibitor), $B C L 2$-associated $\mathrm{X}$ protein (BAX, apoptosis initiator), and Caspase 3 (apoptosis executioner). Rat $A D$ modeling was performed by intrahippocampal injection of amyloid $\beta 1-42$ ( $A \beta 1-42,8 \mu \mathrm{g} / \mathrm{kg})$. Animals were divided into 3 groups of 8 rat: I) control II) AD model III) MSC-treated. Behavioral tests (i.e. Passive Avoidance and Morris Water Maze) showed cognitive improvement, and amelioration of cells in the CA1 area of the hippocampus has been detected by cresyl violet (nissl) staining. Also, real-time polymerase chain reaction (RT-PCR) of the hippocampus indicated an increase in BDNF and NGF genes and a decrease in apoptosis-related genes ( $B C L 2, B A X$, and Caspase 3). Overall, WJ-MSCs improved cognitive functions in $A D$ rat models by increasing neurotrophic factors and decreasing apoptotic factors.

\section{Introduction}

Alzheimer's disease (AD) as the most common cause of dementia in the elderly is associated with memory loss and cognitive-behavioral dysfunctions [1]. These impairments are caused by mainly loss of cholinergic neurons especially in basal forebrain cholinergic neurons (BFCN) as a result of extracellular deposition of $A \beta$ proteins (Senile Plaques) and intracellular formation of neurofibrillary tangles (NFTs) in the brain specifically in the hippocampus region $[2,3]$. It is reported that these lesions (i. e. A $\beta$ deposition and NFTs formation) can be resulted from decreasing in neurotrophic factors mainly brain-derived neurotrophic factor (BDNF) and nerve growth factor (NGF) [4] which is finally led to apoptosis and cell death [5]. Hence, apoptosis, also known as programmed cell death, contributes to neuronal cell death in $A D$ as a result of trophic factor deprivation [6]. The most important key factors involved in apoptosis are the B-cell lymphoma 2 (BCL2) family (mainly anti-apoptotic BCL2 and pro-apoptotic $B A X$ ) and the caspase family (mainly caspase 3 ) $[5,7]$.

Currently, there is no definitive treatment for $A D$, and the current treatments such as administration of acetylcholinesterase inhibitors (AchEl) could just reduce symptoms with probable side effects [8, 9], arising a need for an effective treatment approach.

Recent MSC-based breakthroughs in pre-clinical experiments have been proved more hopeful in the treatment of neurodegenerative diseases [10]. MSCs by having a wide range of capacities including selfrenewal, replenishment of lost cells via differentiation into other cell lineages [11, 12], triggering neurorestorative processes and providing neuroprotection by secretion of trophic factors and antiinflammatory cytokines, decreasing of oxidative stress and apoptosis, stimulating in situ neurogenesis 
[10], are considered a most appropriate choice to treat neurodegenerative diseases [13]. Among all sources of MSCs, recently, the human umbilical cord Wharton's jelly (WJ) has been well considered in MSC-based therapy for neurodegenerative diseases [14] because of being noninvasively accessible and overexpressing high level of neurotrophic factors $[15,16]$. Brain-derived neurotrophic factor (BDNF) and nerve growth factor (NGF) are of the most well-known neuroregulatory factors secreted by WJ-MSCs [16, 17] which have an essential role in neurogenesis, synaptic plasticity, inhibition of apoptosis, immunomodulation, and cell survival [17-19].

Among various routes of cell delivery [20] intranasal (IN) administration seems to be more effective and noninvasive because in this route of cell delivery cells can easily bypass the peripheral organs and bloodbrain barrier (BBB) and migrate along the olfactory nerve into the brain parenchyma and cerebrospinal fluid (CSF) [21, 22].

The aim of this study is to investigate the effects of WJ-MSCs on the cognitive condition in AD rats by evaluation of the levels of neurotrophic factors (BDNF and NGF) and apoptosis-related factors (BCL2, $B A X$, and Caspase 3) after IN application of these cells.

\section{Methods And Materials}

\section{Isolation of MSCs from Wharton's jelly and Confirming of the isolated MSCs}

Stem cell isolation and characterization procedures were performed as described in our previous study [23]. After signing the informed consent form by patients and a witness and approving by Institutional Review Board (IRB), human umbilical cords were collected from full-term cesarean section births at Akbar-Abadi Hospital and transferred to the Cellular and Molecular Research Center at Iran University of Medical Sciences (IUMS), in a sterile specimen container containing $0.9 \%$ normal saline.

Cell culture: Briefly, after washing the cords with phosphate-buffered saline (PBS) and separating the blood vessels and the amniotic membrane, the remaining tissue known as Wharton's Jelly was cut into small pieces of $5 \mathrm{~mm}^{3}$ and transferred into a sterile centrifuge tube containing the enzymes collagenase type I $(300 \mathrm{U} / \mathrm{ml})$ and hyaluronidase $(1 \mathrm{mg} / \mathrm{ml})$ for the first enzymatic digestion putting in an incubator (at $37^{\circ} \mathrm{C}$ in $5 \% \mathrm{CO}_{2}$ ) for $1 \mathrm{~h}$. After filtering the lysed solution with a $70 \mu \mathrm{m}$ cell strainer and centrifuging at $300 \mathrm{~g}$ for $5 \mathrm{~min}$, the remaining tissue was transferred to another centrifuge tube containing $0.1 \%$ trypsin enzyme (Sigma-Aldrich, St Louis, MO, USA) for the second enzymatic digestion for 30 min to isolate cells as much as possible. At the end of the second enzymatic digestion, it was again filtered and centrifuged. Both cell pellets derived from two enzymatic digestion steps were mixed, suspended, and cultured in a complete culture medium included Dulbecco's Modified Eagle's Medium (DMEM; Gibco, Billings, USA) containing 10\% fetal bovine serum (FBS; Sigma, Missouri, USA) and 1\% penicillin-streptomycin antibiotic (Invitrogen, Waltham, USA) for expansion. The viability of the isolated cells was assessed by the trypan blue exclusion method using $0.4 \%$ trypan blue dye (Sigma-Aldrich, St Louis, MO, USA). 
Flow cytometry: To quantitatively detect the mesenchymal CD markers on Wharton's jelly-isolated cells, passage three cells were incubated with monoclonal mouse anti-human antibodies against mesenchymal markers CD105 and CD73 (positive markers) and hematopoietic markers CD45 and CD34 (negative markers) followed by $10 \mathrm{mg} / \mathrm{ml}$ of fluorescein isothiocyanate (FITC)-conjugated anti-mouse immunoglobulin $\mathrm{G}(\mathrm{IgG})$ antibodies (Abcam, Cambridge, UK) for $1 \mathrm{~h}$ at room temperature (RT). A FACS machine (Becton, Dickinson, Franklin Lakes, NJ, USA) and FlowJo software were used for antibody binding analysis and data analysis, respectively.

Immunocytochemistry (ICC): To qualitatively confirm mesenchymal CD markers on Wharton's jellyisolated cells, passage three cells were incubated with primary anti-human nuclei antibodies against mesenchymal markers CD105, CD73 (positive markers), hematopoietic marker CD31 (negative markers), and neuronal marker $\beta$-tubulin III (negative markers). The coverslips were mounted and observed under a Nikon Eclipse TE300 inverted microscope (Spectra Services, Ontario, NY, USA) and images were captured by a CCD camera connected to the microscope.

Osteogenic and Adipogenic Differentiation. Passage three cells were incubated in two differentiation mediums: i) 14 days in the osteogenic induction medium (DMEM-LG plus $10 \% \mathrm{FBS}, 50 \mu \mathrm{g} / \mathrm{ml}$ ascorbate-2 phosphate, $10^{-8} \mathrm{M}$ dexamethasone, and $10 \mathrm{mM}$-glycerophosphate [Invitrogen, Waltham, USA]); and ii) 21 days in the adipogenic differentiation medium (DMEM and $1 \mathrm{~g} / \mathrm{ml}$ glucose [DMEM-LG] plus 10\% FBS, $50 \mu \mathrm{g} / \mathrm{ml}$ of ascorbate- 1 phosphate, $10^{-7} \mathrm{M}$ dexamethasone, and $50 \mu \mathrm{g} / \mathrm{ml}$ indomethacin [Invitrogen, Waltham, USA]). The medium in both induced cell types was changed every 3 days, and completion of cell differentiation was established by morphology and its related staining (i.e., Alizarin Red $\mathrm{S}$ for osteocytes and Oil Red O for adipocytes, [Sigma, Missouri, USA]).

\section{Alzheimer's Disease (AD) Modeling and Intranasal (IN) Administration of WJ-MSCs}

All animal experiments were performed according to the guidelines of the ethical committee of IUMS (IR.iums.rec.1397.1299). Adult 220-260 gr male Wistar rats were obtained from IUMS animal lab and AD modeling was performed as described in our previous study [23]. Briefly, rats were anesthetized using ketamine/xylazine (50/4 mg/kg, i.p), and fixed in the stereotaxic device. After exposing the skull, freshly prepared amyloid $\beta$ 1-42 (Sigma, $8 \mu \mathrm{g} / \mathrm{kg}$ of $A \beta 1-42$ in $16 \mu \mathrm{l}$ PBS) was administered using a hamilton microsyringe during $3 \mathrm{~min}$ into the dorsal hippocampus bilaterally according to Paxinos rat brain atlas (coordinates: $3.6 \mathrm{~mm}$ posterior, $\pm 2 \mathrm{~mm}$ lateral to the bregma, and $3.2 \mathrm{~mm}$ ventral to the skull surface).

The rats were randomly divided into 3 groups ( $\mathrm{n}=8$ in each group) as follows: $\mathrm{i}$ ) control group (vehicle [PBS]-treated rats), ii) AD model group (Aß-treated rats), and iii) MSC-treated group (AD models those which treated with IN administration of WJ-MSCs).

IN-administration of WJ-MSCs was performed on day 14 after AD induction as previously described [21, 24]. Briefly, animals anesthetized and immobilized facing upward. First, $100 \mathrm{U}$ hyaluronidase was freshly dissolved in sterile PBS $(4 \mathrm{U} / \mu \mathrm{l})$ and $3 \mu \mathrm{l}$ of the suspension administered in each nostril using a pipette, which was repeated 4 times up to almost $100 \mathrm{U}$ of hyaluronidase suspension. Next, after keeping treated 
rats facing upward for $30 \mathrm{~min}, 3 \times 10^{5} \mathrm{WJ}-\mathrm{MSC} / \mathrm{rat}$ was suspended in $36 \mu \mathrm{PBS}$ and administered 6 $\mu \mathrm{l} / \mathrm{nostril}$. After $30 \mathrm{~s}$, that the sample drops were completely disappeared the administration with a $2 \mathrm{~min}$ interval repeated 3 times.

\section{Behavioral Evaluations}

Two months after cell therapy, rats were evaluated to assess cognitive functions (learning and memory) performing passive avoidance (PA) response and Morris water maze (MWM) tests.

Passive Avoidance (PA) Response: This test was performed using a shuttle box device with two connected chambers of equal size separated by a guillotine door as previously described [25]. First, a habituation trial was performed to make all animals initially familiar with the environment and apparatus without any stimuli. Next, for the acquisition trial, animals of all groups were guided individually into the illuminated chamber for 10 seconds opening the guillotine door to note the latency to enter the dark chamber as initial latency (IL). After entering the dark chamber, the animals' feet were exposed to electrical stimulation $(0.5 \mathrm{~mA}, 50 \mathrm{~Hz}, 2$ seconds once) through the stainless steel floor in the dark chamber. Finally, after $24 \mathrm{~h}$, for the retention trial, the rats were re-entered into the lighted chamber without any foot shock to record latency to enter the dark chamber as retention time (step-through latency, STL). And total time spent of rats in the dark chamber (time spent in the dark chamber) was also recorded as an indicator of contextual learning. The maximum cut-off time for the STL and time in the dark chamber was 300 and 600 seconds, respectively. If a rat avoided entering the dark chamber for up to 300 seconds, the acquisition of PA response would be considered as successful for that rat.

Morris Water Maze (MWM): Spatial reference learning and memory were evaluated in the water maze task as previously described [26,27]. This test was performed in 6 days including the habituation day (day 1 - apparent platform in the center of tank), acquisition phase (day 2 to 5 - hidden platform in one of the quadrants), and the probe trial stage (day 6 - no platform) using a circular water $\left(22^{\circ} \mathrm{C}\right.$ ) tank that was divided into four imaginary quadrants with a platform as previously described $[28,29]$. In the acquisition phase, the learning process was conducted for 4 days and each day for 4 trials (each trial was set as $60 \mathrm{sec}$.). In the probe trial test on the sixth day, spatial memory was evaluated by removing the hidden platform. In this stage, time spent and distance traveled in the target quadrant (where the platform situated in earlier phases) were measured as two criteria of spatial memory. In both acquisition and probe trial stages, rats' movements were recorded by the camera above the water tank and data collected by a computer equipped with water maze software for analysis.

\section{Histological Evaluations}

RNA extraction and real-time quantitative reverse transcription PCR: Total RNA from hippocampus was extracted and purified with a TRIzol ${ }^{T M}$ (Sigma, Pool, UK) according to manufacturer's instructions, and its concentration was measured with a NanoDrop ND-100 spectrophotometer. Reverse transcription quantitative-polymerase chain reaction (RT-qPCR) was employed to quantify the gene expression levels of neurotrophic factors BDNF and NGF and apoptosis-related factors BCL2, BAX, and Caspase 3 in AD rat 
hippocampus after treatment with WJ-MSCs. 500 nanograms of RNA were reverse transcribed into complementary DNA (cDNA) with the Transcriptor High Fidelity cDNA Synthesis kit (Invitrogen, Paisley, UK) using oligo (dT) primers (Roche). $1 \mu \mathrm{l}$ of the cDNA was amplified using Opticon II (Invitrogen, Paisley, UK) and the SYBR Green PCR Master Mix (Invitrogen, Paisley, UK), following the manufacturer's instructions. PCR was performed in 40 cycles using an annealing temperature of $60^{\circ} \mathrm{C}$ for all genes. Primers used in this study were specifically designed between two adjacent exons (gene runner program) and the sequences have been listed in Table2. mRNA levels for target genes were normalized to reference gene ( $\beta$-actin) by subtracting the $\mathrm{Ct}$ (cycle threshold) value of the reference gene ( $\beta$-actin) from the $\mathrm{Ct}$

value of the samples ( $\Delta \mathrm{Ct}=\mathrm{Ct}$ sample ${ }^{-\mathrm{Ct}}$ reference). The relative expression of the target gene to a calibrator is quantified using $2^{-\Delta \Delta C t}$.

Cresyl Violet (Nissl) Staining: Two months after cell therapy, this test was performed to distinguish healthy neurons from damaged neurons in the cornu ammonis-1 (CA1) area of rats' hippocampus [30]. Briefly, the samples were fixed, $7 \mu \mathrm{m}$ coronal sections ( 5 sections) were taken from -3.84 to -5.8 from Bregma, the sections were transported to gelatinized slides and stained with cresyl violet stain. The images from stained slides were taken by an optical microscope (Carl Zeiss; Oberkochen, Germany) and were averaged using ImageJ software for dark cells in the CA1.

\section{Statistical analyses}

The data were normalized and analyzed using one-way ANOVA followed by Tukey post hoc test to determine the statistical significance between different groups using SPSS software version 24 (SPSS Inc., Chicago, IL, USA). Also, analysis of PA response data was analyzed by paired t-test and a significant difference between groups was determined by one-way ANOVA. All results were considered significant at $P<0.05$ and expressed as mean \pm SEM. Image analysis was done with ImageJ software.

\section{Results}

\section{Wharton's jelly derived mesenchymal stem cells (WJ-MSCs)}

Adherent stem cells were isolated from Wharton's jelly of the human umbilical cord with viability over $95 \%$, which exhibited a spindle-shaped morphology in the primary culture condition after sticking to the bottom of the flask (Fig.1a). Being able to successfully differentiate towards osteocytes by producing calcium nodules (Fig.1b) and towards adipocytes by producing lipid droplets (Fig.1c), which is one of the important characteristics of MSCs, proved WJ-cells mesenchymal type. In quantitative flow cytometry, these cells strongly expressed mesenchymal markers of CD105 and CD73 but did not noticeably express hematopoietic markers of CD34 and CD45 (Fig.2a, and Table1). Also, the results of ICC showed that WJcells were significantly positive for mesenchymal markers CD105 and CD73 and negative for hematopoietic marker CD31 and the neuronal marker $\beta$-tubulin III (Fig.2b).

\section{IN-delivered WJ-MSCs Restored Cognitive Function in Alzheimer Rat Models}


The PA response and Morris Water Maze (MWM) analyses were conducted to evaluate whether WJ-MSCs enhanced spatial learning and memory in A $\beta$-induced Alzheimer's rat models.

Passive Avoidance (PA) Response: The result of the step-through type PA test indicated that initial latency (IL) in entering the dark chamber before applying the electric shock was very low and approximately similar among all animal groups (Fig.3a). However, after an electric shock, the step-through latency (STL) in the $A D$ group was significantly lower than it was in the control group indicating impairment of retention in PA in AD models ( ${ } P<0.05$, Fig.3a). STL was significantly decreased in the MSC-treated group compared with the AD group (\# $P<0.05$, Fig.3a). Moreover, the time spent in the dark chamber after applying electric shock was significantly lower in the MSCs-treated group than it was in the AD group (\# $P$ $<0.05$, Fig.3b). The time spent in the dark chamber after applying electric shock was significantly higher in the $A D$ group than it was in the control group showing impairment of contextual learning in $P A$ in $A D$ models ( $P<0.05$, Fig.3b).

Morris Water Maze (MWM): The findings of MWM were shown in Fig.3c and Fig.3d that respectively represent the distance moved and the time spent in the target quadrant on the probe trial test without platform. After two months of treatment with WJ-MSCs, the rats showed an increased distance moved and also an increased time spent in the target quadrant, which was significantly high compared with AD rats (\# $P<0.05)$. Also, significantly lower distance moved and lower time spent in the target quadrant have been seen in the AD group compared with the control group ( $\left.{ }^{*} P<0.05\right)$. To sum up, in both PA and MWM tests, intranasally delivered WJ-MSCs showed a positive impact on recovering learning and memory in $A D$ rat models.

\section{Gene Expression in Hippocampus}

The mean mRNA levels of neurotrophic factors $B D N F, N G F$, and anti-apoptotic factor $B C L 2$ in the $A D$ model group were significantly low compared with the control group (Fig.4, $*<0.05$ ). After two months of treatment with WJ-MSCs in the MSC-treated group, the level of mRNA of these genes (i. e. BDNF, NGF, and $B C L 2)$ was significantly increased compared with the AD group (\# $P<0.05$ ). With regard to apoptotic factors $B A X$ and Caspase 3 , their mean mRNA level was significantly high in the AD group compared to the control group (Fig.4, ${ }^{*} P<0.05$ ), and they were significantly decreased in the MSC-treated group compared with the AD group (Fig.4, \# $P<0.05$ ).

\section{NissI Stained Cells Were Decreased Following by IN Delivery of WJ-MSCs}

The nissl stained images of the CA1 indicate that the number of dark cells (cells with cell body shrinkage) was considerably higher in the AD group than in the other groups (Fig.5a). The quantitative analysis of the nissl stained images with ImageJ software showed that the number of dark cells was significantly higher in $A D$ than in the control group (Fig.5b, $* P<0.05$ ). Also, the number of dark cells was significantly lower in the treatment group than in the AD group (Fig.5b, \# $P<0.05$ ).

\section{Discussion}


Neurodegeneration and apoptosis play a key role in memory and learning deficits [31, 32]. In this study, intranasally administered human WJ-MSCs in Alzheimer's rat models effectively improved behavioral and cognitive performance by improving memory and learning capabilities. This finding was assessed by measuring the level of trophic factors and apoptosis-related factors in the hippocampus.

In agreement with other studies [24, 33], the IN route of MSC delivery to the brain in this study proved more effective. The possible migration routes of IN administered cells were studied by Danielyan and et. al. in which they hypothesized that these cells could bypass the BBB by migrating from the nasal mucosa through the cribriform plate along the olfactory neural pathway into the brain and cerebrospinal fluid (CSF) [21].

Regarding trophic factors, WJ-MSCs effectively enhanced the trophic support by increasing the levels of $B D N F$ and NGF mRNA in the hippocampus leading to improved cognitive performance in MWM and PA response tests. Similarly, other studies demonstrated that these trophic factors are essential to neural survival. In a study, a clinical trial of NGF gene therapy in AD patients proved useful in preventing cognitive decline [34]. Recently, it was reported that intranasally delivered BMSCs in PD mice could successfully migrate into the hippocampus, olfactory bulb, substantia nigra, striatum, and lateral ventricle and enhance $B D N F$ level in these areas resulting in the survival of existing dopaminergic neurons and finally functional and motor improvement [22]. Also in the aforementioned study, after IN administration of BMSCs, expression of Nestin, a neuronal precursor cell marker, was increased in the subventricular zones (SVZ) indicating stimulation of endogenous neurogenesis [22]. It has been reported that application of fetal human neural stem cells (hNSCs) into the transgenic mice brain, can excrete high quantities of neurotrophic factors including $B D N F$ and $N G F$, and activate the Akt/GSK3 $\beta$ signaling pathway resulting in prevention of tau phosphorylation, attenuation of the synapto-toxic properties of $A \beta$, and promotion of synaptic plasticity and thus enhanced spatial memory [35]. In a study, although NSC transplantation in a transgenic model of $A D$ had no apparent effect on either $A \beta$ or tau, it led to cognitive improvement by elevation of $B D N F$ level and increasing hippocampal synaptic density [36].

Turning to apoptosis subject, there is evidence of apoptosis in the temporal cortex and hippocampus of AD patients [37], and dying neural cells in AD patients' brain showed increased caspase 3 and caspase 6 levels [38]. The current study demonstrated that WJ-MSCs suppressed the apoptosis in A $\beta$-induced AD rat models by enhancing the expression of anti-apoptotic factor $B C L 2$ and suppressing the expression of apoptotic factors $B A X$ and caspase 3. In addition to its neurotrophic action, $B D N F$ has the ability to induce multiple neuroprotective mechanisms including anti-apoptosis (by expression of anti-apoptotic $B C L 2$ protein), anti-oxidation (by expression of anti-oxidative thioredoxin protein), and suppression of autophagy and neuronal death in neurodegenerative diseases [39]. In another study, a substantial decrease in the level of active caspase 3 was found in the brains of hNSC-injected transgenic mice indicating the interfering hNSCs in the prevention of cell death [35]. Beyond apoptosis, inhibition of caspase-3 also effectively blocks microglia activation and neuroinflammation which is common in neurodegenerative diseases including AD and PD [40]. 


\section{Conclusion}

In conclusion, neurotrophic factors play essential roles in neuron survival and alterations in their expression seem to be led to the progression of apoptosis and neurodegeneration. Along with the new drug delivery or gene therapy approaches, MSC-based cell therapy could be considered as the main therapy tool, because MSCs as the secretory of neurotrophic factors can control and support the circumstances by secretion of necessary trophic factors according to the needs of the target organ. The results of this study showed that IN application of human WJ-MSCs in AD rat models can significantly improve learning and memory by enhancing the trophic support and suppressing apoptosis. Neurotrophic support of cholinergic neurons could be resulted from increasing in BDNF and NGF levels in the hippocampus. Also, an increased level of anti-apoptotic factor BCL2 and decreased level of apoptotic factors $B A X$ and Caspase 3 in the hippocampus indicated the suppressed apoptosis in WJ-MSCs treated $A D$ rat models.

\section{Abbreviations}

AD: Alzheimer's disease, MSCs; mesenchymal stem cells, WJ-MSCs: Wharton's jelly-derived MSCs, dexSPIONs: dextran-coated superparamagnetic iron oxide nanoparticles, MTCD: magnetic targeting cell delivery, MRI: Magnetic resonance imaging, ChAT: choline acetyltransferase, AChE: acetylcholinesterase, IL: initial latency, MWM: Morris Water Maze, DMEM: Dulbecco's modified Eagle's medium ,FBS: fetal bovine serum, DLS: dynamic light scattering, BBB: blood brain barrier, TR: Repetition time , FOV: Field of View ,WT :Wild type, IV; intravenous, IV-TC: IV -targeted cells , ICV: intracerebroventricular

\section{Declarations}

Funding: This work was supported by grants from the Iran University of Medical Sciences (IUMS) (97-475-13504) and Iran National Science Foundation (INSF).

Conflict of interest/Competing interests: none.

Availability of data and materials: The datasets used and/or analyzed during the current study are available from the corresponding author on reasonable request.

Code availability: Not applicable

Authors' contributions: $\mathrm{FQH}, \mathrm{EE}, \mathrm{MS}, \mathrm{MA}, \mathrm{RSH}$, and MM collaboratively conducted the experiments, carried out the data analysis, designed the experiments and wrote the manuscript. All authors read and approved the final manuscript.

Ethics approval and consent to participate: All procedures performed in this study were in accordance with the ethical standards of the institutional research ethics committee and with the 1964 Helsinki declaration and its later amendments or comparable ethical standards. Informed consent was obtained 
from all individual participants included in the study. All procedures performed animals were in accordance with the ethical standards of IUMS.

Consent for publication: Not applicable

Acknowledgments

This work was supported by grants from the Iran University of Medical Sciences (IUMS) (97-4-75-13504) and Iran National Science Foundation (INSF) for a Master's dissertation. Experiments were performed at the Cellular and Molecular Research Center (CMRC) and IUMS Core Laboratory (ICL), Tehran, Iran.

\section{References}

1. Madadi, S. and M. Mehdizaded, Alzheimer diseases. Avicenna Journal of Neuro Psych Physiology, 2014. 1(2). http://dx.doi.org/10.17795/ajnpp-19713

2. Barati, E., et al., Effect of Borage on hipocampal TNF-a protein and gene in the Amyloid $\beta$-Peptide (25-35)-Induced of Alzheimer model in rat. Biosciences Biotechnology Research Asia, 2016. 13(1): p. 37-42. http://dx.doi.org/10.13005/bbra/2000

3. Jahanshahi, M., et al., Female rat hippocampal cell density after conditioned place preference. Folia Biologica (Czech Republic), 2014. 60(1): p. 47-51.

4. Sampaio, T.B., et al., Neurotrophic factors in Alzheimer's and Parkinson's diseases: implications for pathogenesis and therapy. Neural regeneration research, 2017. 12(4): p. 549. http://dx.doi.org/10.4103/1673-5374.205084

5. Radi, E., et al., Apoptosis and oxidative stress in neurodegenerative diseases. Journal of Alzheimer's disease, 2014. 42(s3): p. S125-S152. http://dx.doi.org/10.3233/JAD-132738

6. Dragunow, M., et al., Apoptosis, neurotrophic factors and neurodegeneration. Reviews in the Neurosciences, 1997. 8(3-4): p. 223-265. http://dx.doi.org/10.1515/revneuro.1997.8.3-4.223

7. Shaabani, R., et al., Effect of morphine based CPP on the hippocampal astrocytes of male Wistar rats. Asian Journal of Cell Biology, 2011. 6(3): p. 89-96. http://dx.doi.org/10.3923/ajcb.2011.89.96

8. Joyce, N., et al., Mesenchymal stem cells for the treatment of neurodegenerative disease. Regenerative medicine, 2010. 5(6): p. 933-946. http://dx.doi.org/10.2217/rme.10.72

9. Aisen, P.S., J. Cummings, and L.S. Schneider, Symptomatic and nonamyloid/tau based pharmacologic treatment for Alzheimer disease. Cold Spring Harbor perspectives in medicine, 2012. 2(3): p. a006395. http://dx.doi.org/10.1101/cshperspect.a006395

10. Alipour, M., et al., Stem cell therapy in Alzheimer's disease: possible benefits and limiting drawbacks. Molecular biology reports, 2019. 46(1): p. 1425-1446. http://dx.doi.org/10.1007/s11033-018-4499-7

11. Kolf, C.M., E. Cho, and R.S. Tuan, Mesenchymal stromal cells: biology of adult mesenchymal stem cells: regulation of niche, self-renewal and differentiation. Arthritis research \& therapy, 2007. 9(1): p. 204. http://dx.doi.org/10.1186/ar2116 
12. Yang, H., et al., Concise Review: The Regulatory Mechanism of Lysine Acetylation in Mesenchymal Stem Cell Differentiation. Stem Cells International, 2020. 2020.

https://doi.org/10.1155/2020/7618506

13. Lo Furno, D., G. Mannino, and R. Giuffrida, Functional role of mesenchymal stem cells in the treatment of chronic neurodegenerative diseases. Journal of cellular physiology, 2018. 233(5): $p$. 3982-3999. http://dx.doi.org/10.1002/jcp.26192

14. Lee, N.K., et al., Agouti related peptide secreted via human mesenchymal stem cells upregulates proteasome activity in an Alzheimer's disease model. Scientific reports, 2017. 7(1): p. 1-9. http://dx.doi.org/10.1038/srep39340

15. Donders, R., et al., Human Wharton's Jelly-Derived Stem Cells Display a Distinct Immunomodulatory and Proregenerative Transcriptional Signature Compared to Bone Marrow-Derived Stem Cells. Stem cells and development, 2018. 27(2): p. 65-84. http://dx.doi.org/10.1089/scd.20170029

16. Ribeiro, C.A., et al., The secretome of stem cells isolated from the adipose tissue and Wharton jelly acts differently on central nervous system derived cell populations. Stem cell research \& therapy, 2012. 3(3): p. 18. https://doi.org/10.1155/2014/438352

17. Hsieh, J.-Y., et al., Mesenchymal stem cells from human umbilical cord express preferentially secreted factors related to neuroprotection, neurogenesis, and angiogenesis. PloS one, 2013. 8(8). https://doi.org/10.1371/journal.pone.0072604

18. Mattson, M.P., S. Maudsley, and B. Martin, BDNF and 5-HT: a dynamic duo in age-related neuronal plasticity and neurodegenerative disorders. Trends in neurosciences, 2004. 27(10): p. 589-594. https://doi.org/10.1016/j.tins.2004.08.001

19. Teixeira, F.G., et al., Mesenchymal stem cells secretome: a new paradigm for central nervous system regeneration? Cellular and Molecular Life Sciences, 2013. 70(20): p. 3871-3882. https://doi.org/10.1007/s00018-013-1290-8

20. Park, S.E., et al., Optimal mesenchymal stem cell delivery routes to enhance neurogenesis for the treatment of Alzheimer's disease. Histology and histopathology: cellular and molecular biology, 2018. 33(6): p. 533-541. https://doi.org/10.14670/HH-11-950

21. Danielyan, L., et al., Intranasal delivery of cells to the brain. European journal of cell biology, 2009. 88(6): p. 315-324. https://doi.org/10.1016/jejcb.2009.02.001

22. Tang, Y., et al., Intranasal Delivery of Bone Marrow Stromal Cells Preconditioned with Fasudil to Treat a Mouse Model of Parkinson's Disease. Neuropsychiatric Disease and Treatment, 2020. 16: p. 249. https://doi.org/10.2147/NDT.S238646

23. Hour, F.Q., et al., Magnetic targeted delivery of the SPIONs-labeled mesenchymal stem cells derived from human Wharton's jelly in Alzheimer's rat models. Journal of Controlled Release, 2020. https://doi.org/10.1016/j.jconrel.2020.02.035

24. Shahror, R.A., et al., Tracking Superparamagnetic Iron Oxide-labeled Mesenchymal Stem Cells using MRI after Intranasal Delivery in a Traumatic Brain Injury Murine Model. JoVE (Journal of Visualized Experiments), 2019(153): p. e60450. https://doi.org/10.3791/60450. 
25. Vafaee, F., et al., Effect of Recombinant Insulin-like Growth Factor-2 Injected into the Hippocampus on Memory Impairment Following Hippocampal Intracerebral Hemorrhage in Rats. Galen Medical Journal, 2018. 7: p. 1353. https://doi.org/10.22086/gmj.v0i0.1353

26. Morris, R.G., et al., Place navigation impaired in rats with hippocampal lesions. Nature, 1982. 297(5868): p. 681-683. https://doi.org/10.1038/297681a0

27. Mehdizadeh, M., et al., Protective effects of cyperus rotundus extract on amyloid $\beta$-peptide (1-40)induced memory impairment in male rats: a behavioral study. Basic and clinical neuroscience, 2017. 8(3): p. 249. https://doi.org/10.18869/nirp.bcn.8.3.249

28. Morris, R., Developments of a water-maze procedure for studying spatial learning in the rat. Journal of neuroscience methods, 1984. 11(1): p. 47-60. https://doi.org/10.1016/0165-0270(84)90007-4

29. Tamtaji, O.R., et al., Protective Effects of Red Onion (Allium cepa) Ethanolic Extract on Learning and Memory Impairments in Animal Models of Diabetes. Galen Medical Journal, 2017. 6(3): p. 249-257. https://doi.org/10.22086/gmj.v6i3.909

30. Faghani, M., et al., The Neuroprotective Effect of Atorvastatin on Apoptosis of Hippocampus Following Transient Global Ischemia/Reperfusion. Galen Medical Journal, 2016. 5(2): p. 82-89.

31. Wozniak, D.F., et al., Apoptotic neurodegeneration induced by ethanol in neonatal mice is associated with profound learning/memory deficits in juveniles followed by progressive functional recovery in adults. Neurobiology of disease, 2004. 17(3): p. 403-414. https://doi.org/10.1016/j.nbd.2004.08.006

32. Mattson, M.P., Apoptosis in neurodegenerative disorders. Nature reviews Molecular cell biology, 2000. 1(2): p. 120-130. https://doi.org/10.1038/35040009

33. Beigi Boroujeni, F., et al., Intranasal delivery of SDF-1a-preconditioned bone marrow mesenchymal cells improves remyelination in the cuprizone-induced mouse model of multiple sclerosis. Cell biology international, 2020. 44(2): p. 499-511. https://doi.org/10.1002/cbin.11250

34. Tuszynski, M.H., et al., A phase 1 clinical trial of nerve growth factor gene therapy for Alzheimer disease. Nature medicine, 2005. 11(5): p. 551-555. https://doi.org/10.1038/nm1239

35. Lee, I.-S., et al., Human neural stem cells alleviate Alzheimer-like pathology in a mouse model. Molecular neurodegeneration, 2015. 10(1): p. 38. https://doi.org/10.1186/s13024-015-0035-6

36. Blurton-Jones, M., et al., Neural stem cells improve cognition via BDNF in a transgenic model of Alzheimer disease. Proceedings of the National Academy of Sciences, 2009. 106(32): p. 1359413599. https://doi.org/10.1073/pnas.0901402106

37. Dragunow, M., et al., In situevidence for DNA fragmentation in Huntington's disease striatum and Alzheimer's disease temporal lobes. Neuroreport, 1995. 6(7): p. 1053-1057. https://doi.org/10.1097/00001756-199505090-00026

38. Deigner, H.-P., U. Haberkorn, and R. Kinscherf, Apoptosis modulators in the therapy of neurodegenerative diseases. Expert opinion on investigational drugs, 2000. 9(4): p. 747-764. https://doi.org/10.1517/13543784.9.4.747

39. Chen, S.-D., et al., More insight into BDNF against neurodegeneration: anti-apoptosis, anti-oxidation, and suppression of autophagy. International journal of molecular sciences, 2017. 18(3): p. 545. 
https://doi.org/10.3390/ijms18030545

40. D'Amelio, M., M. Sheng, and F. Cecconi, Caspase-3 in the central nervous system: beyond apoptosis. Trends in neurosciences, 2012. 35(11): p. 700-709. https://doi.org/10.1016/j.tins.2012.06.004

\section{Tables}

Table1 Flow cytometry analysis of Wharton's jelly-derived stem cells. WJ cells strongly expressed mesenchymal markers CD105 and CD73 and weakly expressed hematopoietic markers CD34 and CD45

\section{Flowcytometery analysis}

Cell surface markers

Rate of WJ-MSCs which express CD marker Mean $\pm \operatorname{sem}(\%)$

Mesenchymal markers

CD105 and CD73

$97.00 \pm 1.96$

Hematopoitic markers

CD34 and CD45

$4.19 \pm 1.42$

Table2 Sequence of specific primers used for quantitative real-time revers transcription PCR 


\begin{tabular}{|c|c|c|}
\hline Gene Name & & Primer Sequence \\
\hline \multirow[t]{2}{*}{ NGF } & Forward & 5'-CATCGCTCTCCTTCACAG-3' \\
\hline & Revers & 5'-TAGAACAACATGGACATTACG-3' \\
\hline \multirow[t]{2}{*}{$B D N F$} & Forward & 5'-TTCTGTAATCGCCAAGGT-3' \\
\hline & Revers & 5'-TGGTCATCACTCTTCTCA-3' \\
\hline \multirow[t]{2}{*}{$B A X$} & Forward & 5'-CTGGGATGAATGGGGGAAGGGGAAA-3' \\
\hline & Revers & 5'-AAAGGGGACCTGAGGTTTATTGGCG-3' \\
\hline \multirow[t]{2}{*}{ BCL2 } & Forward & 5'-ATGGCGCAAGCCGGGAGAAC-3' \\
\hline & Revers & 5'-CGCGTCCGCATCTCCAGCAT-3' \\
\hline \multirow[t]{2}{*}{ Caspase 3} & Forward & 5'-CTCTGGTACGGATGTGGACG-3' \\
\hline & Revers & 5'-ССССТTCATCACCATGGCTT-3' \\
\hline \multirow[t]{2}{*}{$\beta$-actin } & Forward & 5'-TCTATCCTGGCCTCACTGTC-3' \\
\hline & Revers & 5'-AACGCAGCTCAGTAACAGTCC-3' \\
\hline
\end{tabular}

\section{Figures}

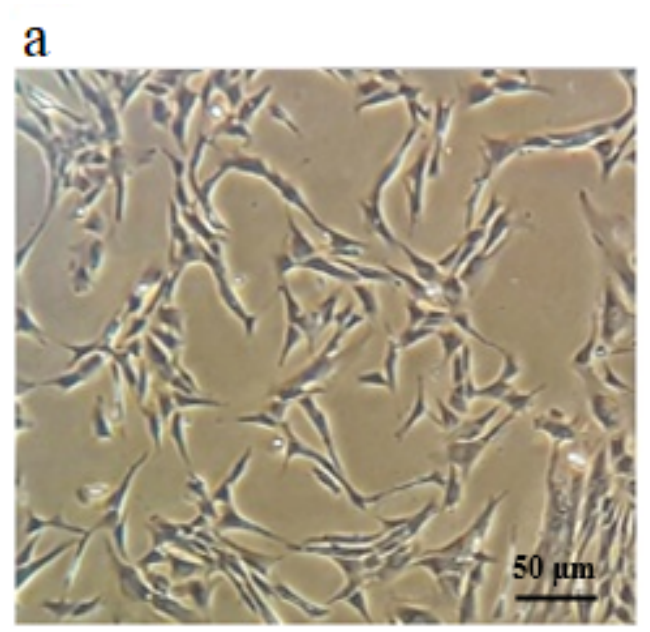

b

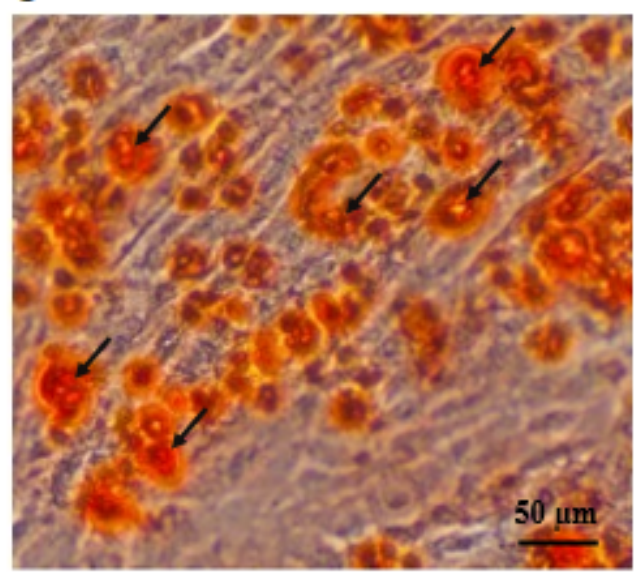

c

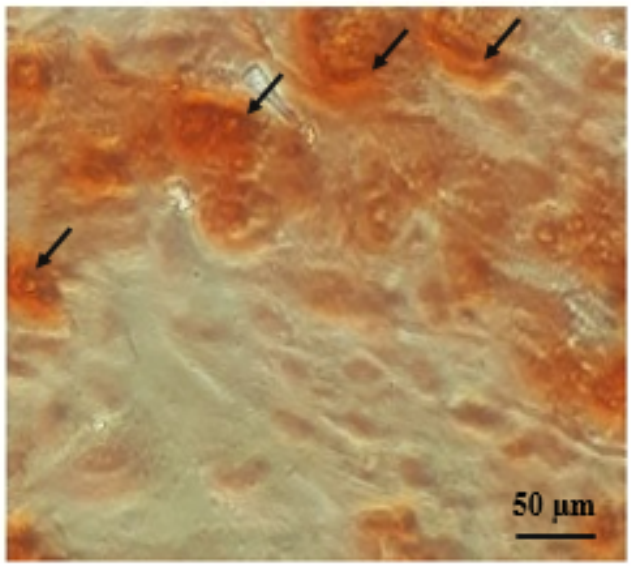




\section{Figure 1}

Human Wharton's jelly-derived cells (WJ-cells) and their in vitro differentiation towards other cell lineage types. a) The cells isolated from Wharton's jelly are adherent with a spindle-shaped chondrocyte-like phenotype. Differentiation into osteocytes (b) and adipocytes (c) caused a considerable amount of calcium nodules (arrows in b) and lipid droplets (arrows in c)
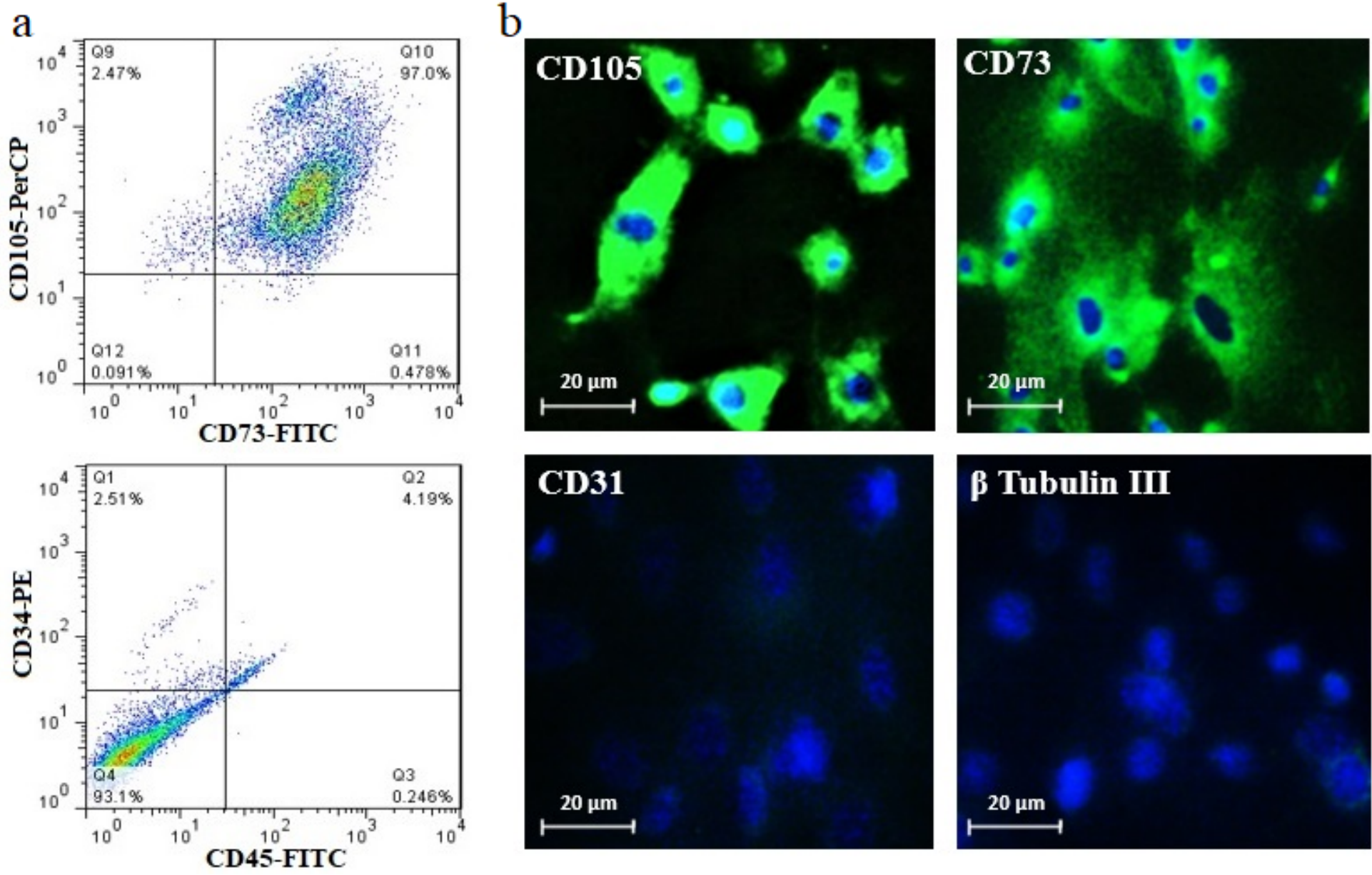

\section{B Tubulin III}

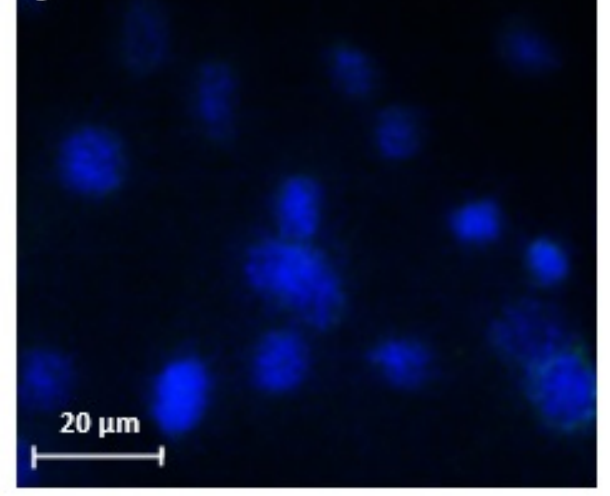

\section{Figure 2}

a) Flow cytometry analysis showed that a large amount of WJ-SCs was positive for both CD105 and CD73 mesenchymal markers, while just a small amount of them expressed the hematopoietic markers of CD34 and CD45. b) Immunocytochemistry (ICC) analysis showed that the CD105 and CD73 mesenchymal markers are largely expressed in WJ-derived stem cells and came in green with FITC. Hematopoietic marker of CD31 and neuronal marker of $\beta$ tubulin III are weakly expressed by these cells. Blue color: nuclei of cells stained by DAPI 
a
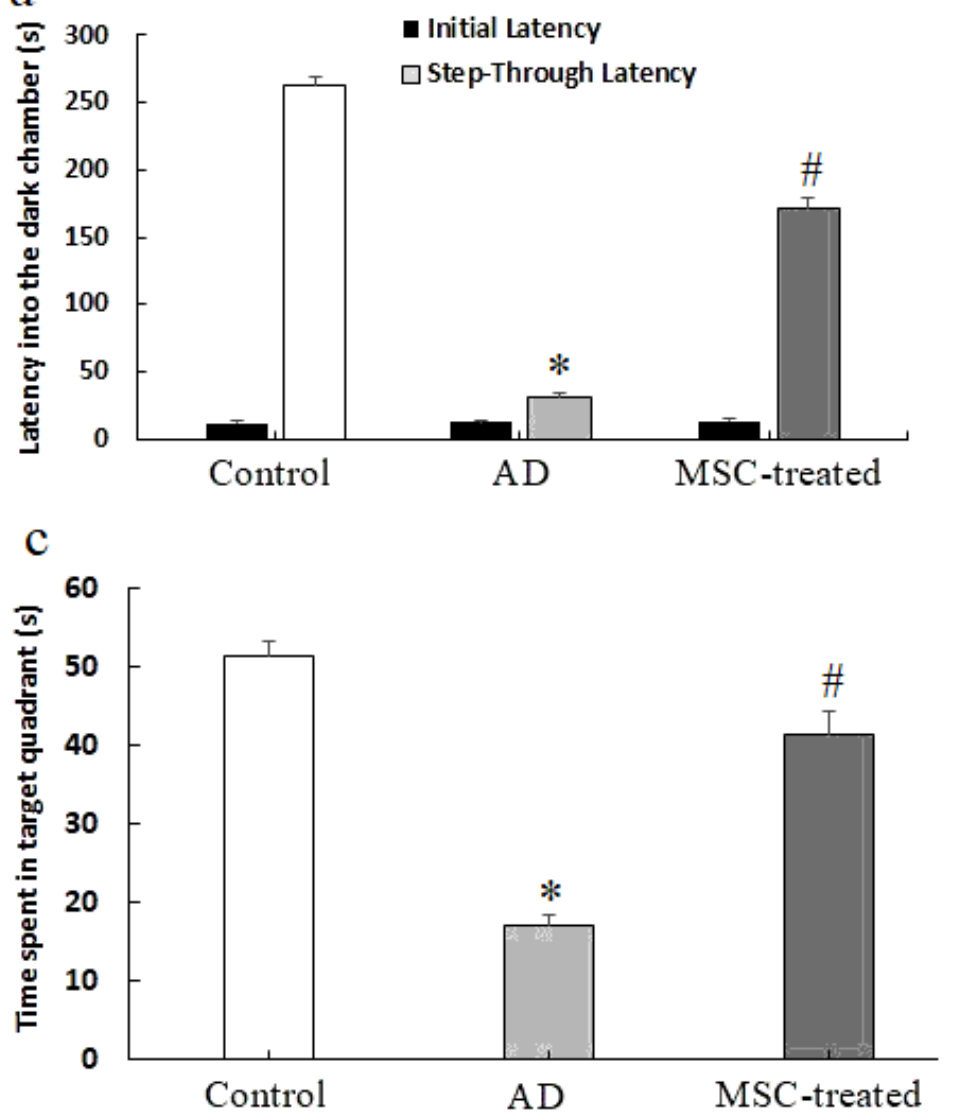

b

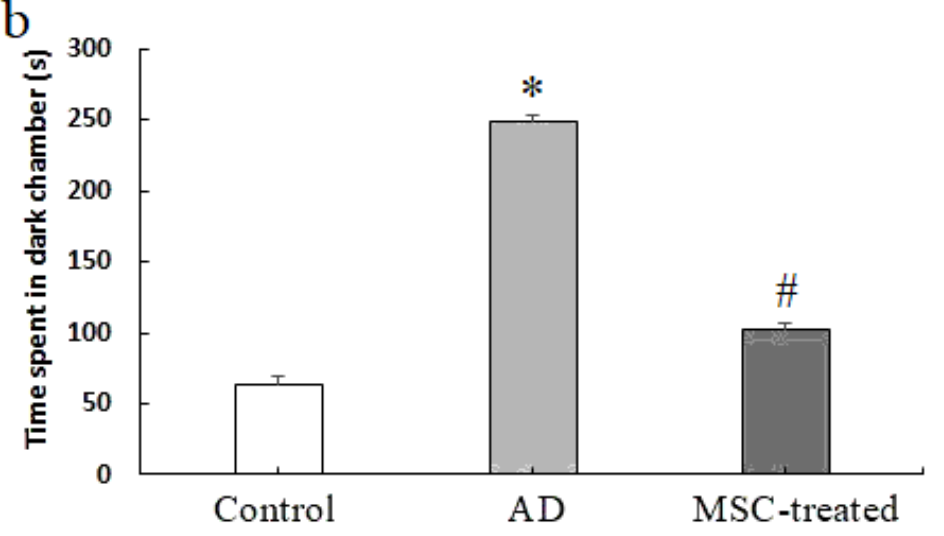

d

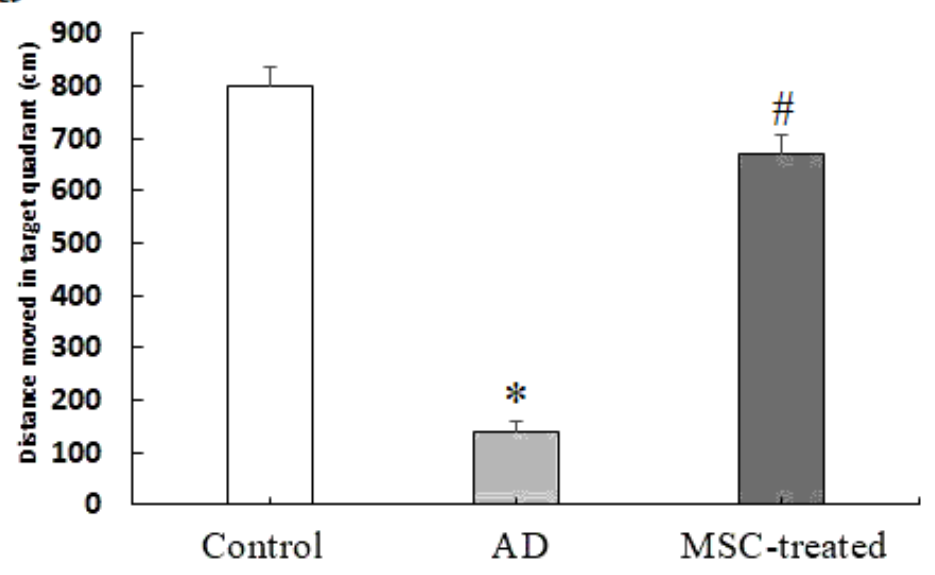

Figure 3

Behavioural evaluations. Passive avoidance (PA) response test: a) latency in entering into the dark chamber before [initial latency (IL)] and after [step-through latency (STL)] the electric shock. IL was very low and almost similar among all animal groups. The WJ-MSCs-treated group showed an increased STL after two months of treatment which was significantly higher than it was in the AD group (\# $P<0.05$ ). $S T L$ was significantly lower in the AD group than it was in the control group $(* P<0.05)$. b) time spent in the dark chamber after applying electric shock was significantly lower in the WJ-MSCs treated group than in the AD group (\# $P<0.05$ ) and significantly higher in the AD group than in the control group (* $P<0.05$ ). Morris Water Maze (MWM) test: $\mathrm{c}$ ) time spent and d) distance moved in the target quadrant. The WJMSCs-treated rats showed an increased distance moved and also an increased time spent in the target quadrant, after two months of treatment which was significantly high compared with $A D$ rats $(\# P<0.05)$. These items (distance moved and time spent in the target quadrant) was significantly low in $A D$ rats compared with the control group $(* P<0.05)$ 

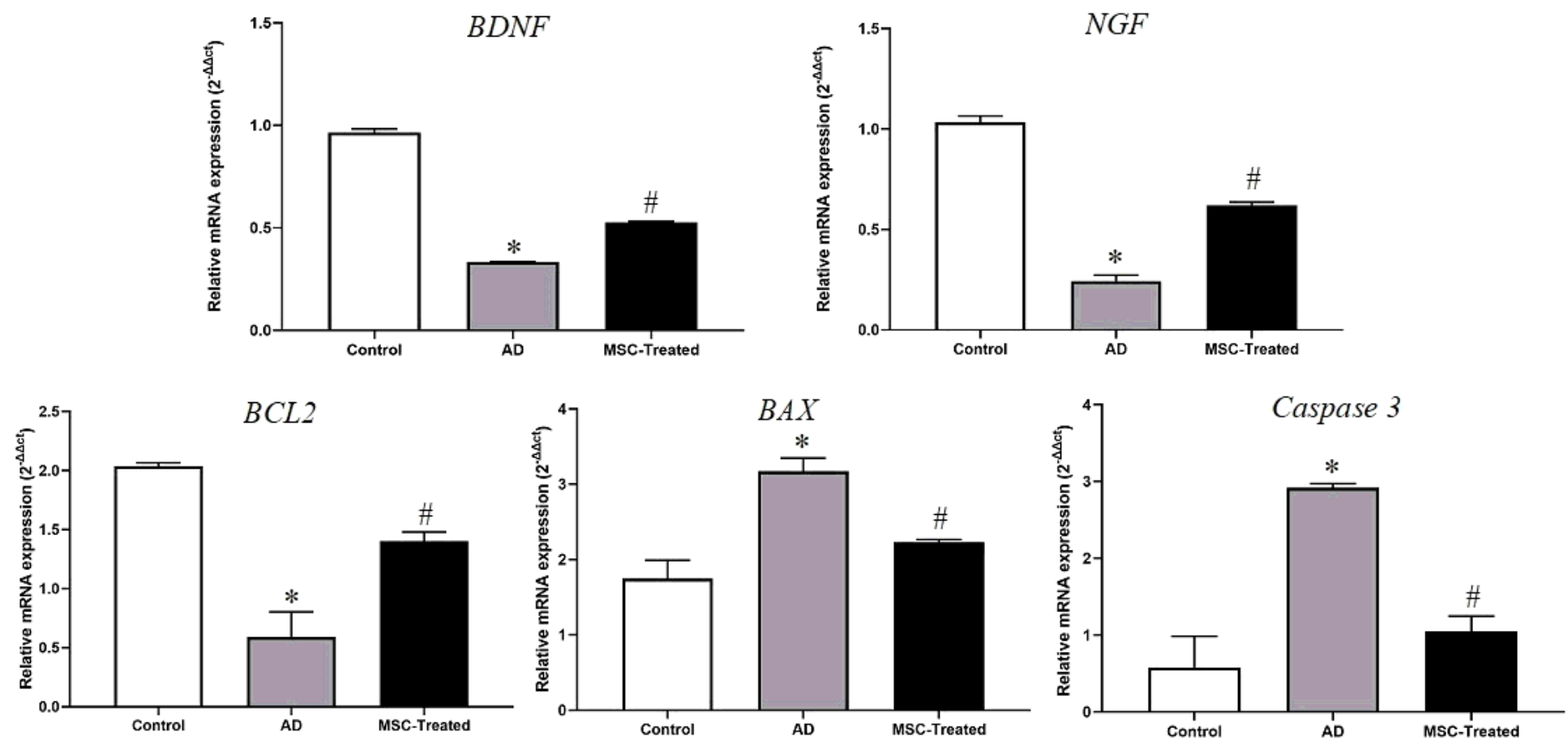

Figure 4

Evaluation of mRNA levels gene expression by RT-qPCR in the hippocampus. Data are represented as mean $\pm S E M$. $\beta$-actin was used as an internal housekeeping control. The mean mRNA level of neurotrophic factors NGF, BDNF, and anti-apoptotic factor BCL2 in the AD group was significantly low compared with the control group ( $\mathrm{P}<0.05$ ). After two months of treatment with WJ-MSCs in the MSCtreated group, these level of mRNA (NGF, BDNF, and BCL2) was significantly increased compared with the $A D$ group (\# $P<0.05$ ). With regard to apoptotic factors $B A X$ and Caspase 3 , their mean mRNA level was significantly high in the $A D$ group compared with the control group ( $\left.{ }^{*}<0.05\right)$, and they were significantly decreased in the MSC-treated group compared with the AD model group $(\# P<0.05)$ 

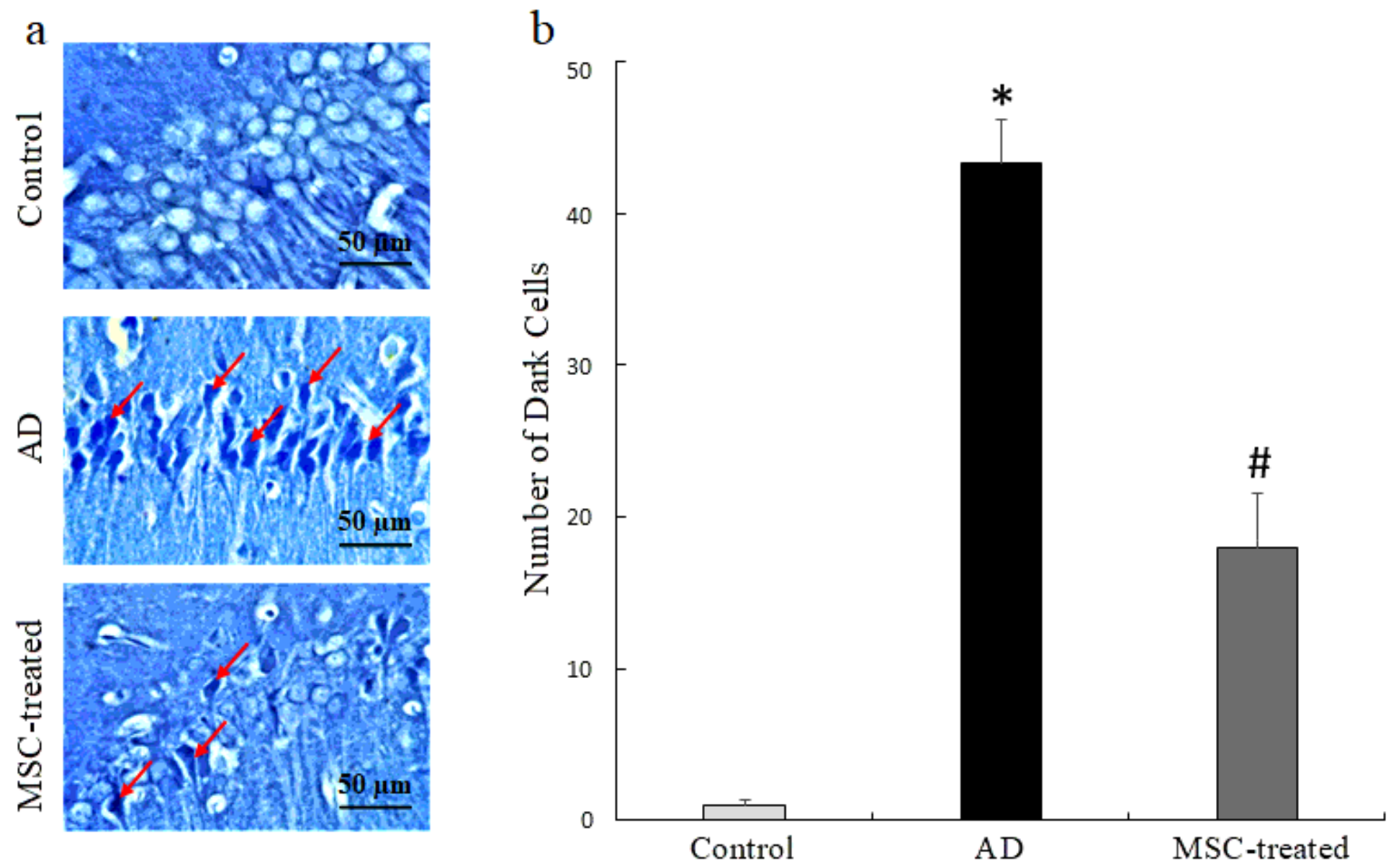

\section{Figure 5}

a) Nissl staining of CA1 region of the hippocampus in which red arrows show the nissl-stained dead or dark cell. b) The quantitative analysis showed that the number of nissl-stained dark cells was much more in the CA1 area of the hippocampus of the AD model group ( $\mathrm{P}<0.05$ compared with the control group). After two months of WJ-MSCs treatment, the number of dark cells was significantly decreased compared with the AD group $(\# P<0.05)$

\section{Supplementary Files}

This is a list of supplementary files associated with this preprint. Click to download.

- GraphicalAbstarct.png 